\title{
INJECTING ROOMS IN SWITZERLAND
}

\section{Kate Dolan}

Research Fellow

National Drug and Alcohol Research Centre, UNSW

This report describes injecting rooms in Switzerland, and discusses the history of injecting rooms, the context in which they began, and how they operate. These are observations made during a visit to Switzerland in February 1996, and from discussions with health workers and researchers in Switzerland.

The main aim of injecting rooms in Switzerland is to reduce the public health risks, and the public nuisance, associated with drug injecting. These health risks include death from overdose, and infection with blood-borne viruses (HIV, HBV and HCV) from using shared injecting equipment. Medically-trained staff are available at all times to resuscitate clients who experience drug overdoses on the premises.

\section{CONTEXT FOR INJECTING ROOMS}

Switzerland has implemented a number of innovative HIV prevention programs that have rarely been adopted elsewhere. Examples include 'needle parks'—open drug scenes where drug users buy, sell and use drugs; a heroin trial that included current prisoners; and syringe exchange schemes for prisoners. Switzerland has a high number of injecting drug users (IDUs), and they are very visible in the streets because of housing shortages. HIV prevalence among Swiss IDUs was already high, up to 50 per cent in some cities, when HIV-AIDS was first recognised.

The first injecting room in Switzerland was established in Bern in 1986 after health workers noticed that IDUs were marginalised from mainstream society, and were being shunned from cafes and restaurants. These health workers proposed establishing a special cafe for IDUs within a government-run health centre that could be a convenient point of contact with IDUs who did not utilise health services. Another factor was the desire to reduce public nuisance resulting from IDUs injecting in parks and public toilets. IDUs then began injecting in these cafes. The workers soon realised that this provided an opportunity to monitor and modify IDUs' risk behaviour to reduce harms associated with injecting. Community opposition in Basel decreased after concerned residents attended an open day at the centre.

\section{THE CENTRES AND INJECTING ROOMS}

The injecting rooms are housed within centres, which also contain a cafe, a counselling room and a clinic for primary medical care. The injecting rooms are discrete rooms within the centres, and the terms 'centre' and 'injecting room' will be used in this report to distinguish between the two levels of intervention.

The actual injecting rooms are small and have a sterile ambience. The three rooms visited contain three tables where up to six clients could sit to prepare and inject their drugs. Injecting paraphernalia such as needles and syringes, a candle, sterile water and spoons, were placed at each position at the tables. Paper towels, cotton pads, bandaids, and rubbish bins were also provided. The tabletops were made of stainless steel for ease of cleaning.

\section{Access to the injecting rooms}

Staff must verify that clients are at least 16 years old and have a history of injecting before they are allowed to use the injecting rooms. Each room has a maximum capacity that varies depending on its size.

In addition to rules that are common in most drug agencies — such as no violence or drug dealing - there are specific rules for the injecting rooms. Clients must wash their hands on entering the injecting room, and clean their own place at the table after injecting. Clients are not allowed to smoke in the injecting rooms. Most centres have a maximum time limit ( 30 or 60 minutes) that a client can spend in the injecting room. Clients are allowed to prepare only their own drugs in the injecting room. Staff are not permitted to help clients inject in any centre. Clients who break the rules are barred from the centre for a few days.

\section{Staff and operation}

At all centres at least one staff member is present in the injecting rooms at all times. This staff member changes every hour or so, because extended periods in the injecting room are considered to be too taxing. All staff are trained to resuscitate clients if they overdose, although one staff member has the prime responsibility for this duty.

Most centres open for approximately seven hours a day, and some centres are closed for one or two days a week. Centres usually operate at full capacity. In cities with a number of centres, operating times are staggered to maximise the number of hours per day that IDUs can inject safely. Doctors are employed on a sessional basis to visit a centre for a few hours a week. Some centres have direct phone lines to the police and ambulance service.

\section{In the event of an overdose}

When a client collapses, the worker in the injecting room calls another worker to assist. Oxygen from a small bottle is administered to the client via a facemask and simple resuscitation bag until the client regains consciousness. 
If the client doesn't resume breathing within 10 minutes, an ambulance is called. Naloxone, an antagonist commonly used to reverse the effects of narcotics, is not used to revive clients.

\section{Other services}

Counselling, referral to drug treatment, free soup, tea and coffee, and cheap fruit and vegetables are provided in the centres. In Zurich clients volunteer to work in the cafe, and to collect discarded syringes in the vicinity of the centre.

\section{RESEARCH INTO INJECTING ROOMS}

Approximately 100 clients a day visit the centres in Zurich and Basel. Within a one-year period in three centres in Zurich, there were an estimated 68,000 injections, 3,000 abscesses treated, 22 clients resuscitated and 10 calls for an ambulance to attend. A comparison of clients surveyed in Bern in 1990 and in 1995, undertaken by the University of Basel, showed a significant increase in the proportion of clients reporting that their first injection with a sterile needle and syringe occurred during the study period. Reuse of injecting equipment decreased significantly during the study.

The main reasons given for attending injecting rooms in 1995 were: to inject in peace ( 86 per cent); to obtain free injecting equipment (33 per cent); and to have medical attention available. There have been no deaths in any injecting rooms in Switzerland to date. Workers in Basel believe that the number of deaths due to overdose in the community has decreased as a result of injecting rooms.
In Bern, workers believe they have made the injecting ritual less dangerous by moving clients from $2 \mathrm{ml}$ to $1 \mathrm{ml}$ syringes, which carry less risk of blood-borne infection.

Injecting rooms provide drug users with hygienic and controlled conditions; prevent infection by providing sterile syringes, needles and condoms; give access to medical care; and provide opportunities for intervention in the case of such emergencies as overdoses. All key stakeholders have overwhelmingly welcomed the demonstration project and, in Basel, the evaluation team has called for the continuance of the strategy to provide a stable environment for Basel IDUs. ${ }^{1}$

\section{CONCLUSIONS}

Injecting rooms are only needed in areas of cities with high injecting drug use and frequent public injection. The main benefits of injecting rooms have been the reduction of public nuisance and the improvement of health in a very vulnerable and unhealthy group. Injecting rooms enable the adoption of less hazardous injecting practices, reduce the number of overdose deaths, minimise the nuisance to the community of injecting in public places, and probably reduce HIV transmission. The centres are well tolerated in Swiss communities. Some IDUs have entered treatment as a result of attending injecting rooms.

\section{REFERENCE}

1. Ronco C, Spuler G, Coda P, Schopfer R. Evaluation der Gassenzimmer I, II and III in Basel. Basel: Institut fur Sozial und Praventivmedizin der Universitat Basel, 1994. Unpublished. Fit

\section{AN INJECTING ROOM FOR KINGS CROSS}

As part of the NSW Government response to the Drug Summit, it was announced that the Sisters of Charity Health Service, and St Vincent's Hospital Darlinghurst, had reached agreement with the NSW Government to run an 18-month clinical trial of a medically-supervised injecting room. The initial proposal is for a facility to be located in Kings Cross and operate for seven hours per day, every day. The facility will be staffed by a medical director, registered nurses, counsellors and security staff. Necessary amendments to the Drug Misuse and Trafficking Act are expected to be made in the spring session of Parliament. Final approval rests with the DirectorGeneral of Health and the Police Commissioner. An independent evaulation team has been identified. 